\title{
Effects of changing scale on landscape pattern analysis: scaling relations
}

\author{
Jianguo $\mathrm{Wu}$ \\ Landscape Ecology and Modeling Laboratory (LEML), Faculty of Ecology, Evolution, and Environmental \\ Science, School of Life Sciences, Arizona State University, Tempe, AZ 85287-1601, USA; (e-mail: \\ Jingle.Wu@asu.edu)
}

Received 2 December 2002; accepted in revised form 20 August 2003

Key words: Landscape metrics, Pattern analysis, Scale effects, Scaling, Scalograms, Grain, Extent

\begin{abstract}
Landscape pattern is spatially correlated and scale-dependent. Thus, understanding landscape structure and functioning requires multiscale information, and scaling functions are the most precise and concise way of quantifying multiscale characteristics explicitly. The major objective of this study was to explore if there are any scaling relations for landscape pattern when it is measured over a range of scales (grain size and extent). The results showed that the responses of landscape metrics to changing scale fell into two categories when computed at the class level (i.e., for individual land cover types): simple scaling functions and unpredictable behavior. Similarly, three categories were found at the landscape level, with the third being staircase pattern, in a previous study when all land cover types were combined together. In general, scaling relations were more variable at the class level than at the landscape level, and more consistent and predictable with changing grain size than with changing extent at both levels. Considering that the landscapes under study were quite diverse in terms of both composition and configuration, these results seem robust. This study highlights the need for multiscale analysis in order to adequately characterize and monitor landscape heterogeneity, and provides insights into the scaling of landscape patterns.
\end{abstract}

\section{Introduction}

Spatial heterogeneity is ubiquitous across all scales and forms the fundamental basis of the structure and functioning of landscapes, be they natural or cultural. To understand how landscapes affect, and are affected by, biophysical and socioeconomic activities, we must be able to quantify spatial heterogeneity and its scale dependence (i.e., how patterns change with scale). Indeed, much of what has been done in geography, remote sensing and ecology has to do with describing, manipulating, and understanding spatial heterogeneity. With the increasing recognition of the importance of spatial heterogeneity by ecologists in the past two decades, landscape ecology has come of age with a distinctive emphasis on the spatial dimension of ecological pattern and process (Turner et al.
2001; Wu and Hobbs 2002). An important unifying concept in dealing with heterogeneity and integrating ecological and geographical sciences is scale. While the term "scale" may refer to any one or combinations of several concepts, including grain (or resolution, support), extent, lag (or spacing), and cartographic ratio (Wiens 1989; Lam and Quatrochi 1992; Schneider 2001; Dungan et al. 2002), in this paper it refers only to "grain" (the spatial resolution of a map) and "extent" (the map size, or "geographic scale" as defined by Lam and Quatrochi 1992).

The scale dependence of spatial heterogeneity has been recognized in both geography and ecology for decades. Two different but related connotations of scale dependence of spatial heterogeneity may be distinguished. The first implies that spatial heterogeneity exhibits various patterns at different scales, or 
patterns have distinctive "operational" scales (sensu Lam and Quattrochi 1992) at which they can be best characterized. Apparently, this perspective is consistent with the concepts of characteristic scale and hierarchy that have been prevalent in ecological literature since the 1980s (Allen and Starr 1982; Allen et al. 1984; O'Neill et al. 1986; Urban et al. 1987; $\mathrm{Wu}$ and Loucks 1995; Wu 1999). The second connotation refers to the dependence of observed spatial heterogeneity on the scale of observation and analysis - often discussed in terms of scale effects on image classification and spatial pattern analysis. Scale effects have long been studied in human geography as part of the modifiable areal unit problem or MAUP - the problem in spatial analysis that occurs when area-based data are aggregated (Openshaw 1984; Arbia et al. 1996; Jelinski and Wu 1996; Wrigley et al. 1996; Marceau 1999). MAUP includes two distinct but related aspects: the result of statistical analysis is affected by both the level of data aggregation or grain size (so-called "scale problem") and by alternative ways of aggregating pixels at a given grain size (often called the "zoning problem" or "aggregation problem"). MAUP has frequently been discussed together with the so-called "ecological fallacy" (sensu Robinson 1950) which refers to inappropriate extrapolation of statistical relationships from one scale to another. Unfortunately, the term "ecological fallacy" is misleading and can be irritating because the problem it refers to occurs across all natural and social sciences whenever heterogeneity and nonlinearity exist and because the use of the term "ecological" here is not at all scientifically rigorous. A more appropriate term for this kind of scale-related problems may be "spatial transmutation" (sensu O'Neill 1979; also see King et al. 1991; Wu and Levin 1994).

Scale effects on spatial pattern analysis may occur in each of the following three situations: (1) changing grain size (or resolution) only, (2) changing extent only, and (3) changing both grain and extent. As noted earlier, the modifiable areal unit problem involves both the effect of altered grain size and the way of this alteration. Similarly, there are also different ways of changing extent: e.g., boxing out from the center of a map or starting from one corner along a diagonal direction. In general, much more research has been done into the effects of changing grain size (particularly in the context of MAUP) than those of changing extent, and a quantitative understanding of these two kinds of scale effects across different systems and methods is still lacking. Scale effects do not necessarily have to be considered as problems because they can be used for understanding the multiple-scale characteristics of landscapes (Jelinski and Wu 1996; Wu et al. 2000; Wu et al. 2003). In principle, the relevant pattern is revealed only when the scale of analysis approaches the operational scale of the phenomenon under study (Allen et al. 1984; Wu and Loucks 1995; Wu 1999). In practice, however, not all scale breaks revealed in multiscale analysis by resampling data correspond to actual operational scales or hierarchical levels due to inaccuracies caused by the methods of data aggregation and analysis (Wu et al. 2000; Hay et al. 2001).

While most of the MAUP studies prior to the 1990's focused on traditional statistical measures (e.g., mean, variance, regression and correlation coefficients) and spatial interaction models, scale effects have been increasingly studied using landscape metrics (or indices) in ecology, remote sensing, and geography in the past two decades (Meentemeyer and Box 1987; Turner et al. 1989; Turner et al. 2001; Bian and Walsh 1993; Moody and Woodcock 1994; Benson and Mackenzie 1995; Wickham and Riitters 1995; Jelinski and Wu 1996; O’Neill et al. 1996; Qi and $\mathrm{Wu}$ 1996; Wu et al. 2003). These studies have shed new light on the problems of scale effects in pattern analysis as well as the multiscaled nature of spatial heterogeneity. Yet, most of the existing studies that used landscape metrics considered only a few indices with a narrow range of scales, and few have gone beyond merely reporting the existence of scale effects to explore their generalities across different landscapes. Thus, although ecologists are well aware that changing scale often affects landscape metrics, scaling relations are yet to be developed.

To systematically explore the effects of changing scale on pattern analysis, using simulated landscapes with known structural characteristics is both necessary and effective (Gardner et al. 1987; Amrhein 1995; Arbia et al. 1996; Hargis et al. 1998; Wu et al. 2000; Saura and Martinez-Millan 2001). However, comprehensive empirical studies using real landscape data are needed because only such studies can tell us what kinds of scaling relations may exist and how variable or consistent they are in actual (not just simulated) landscapes. Such information is indispensable for more in-depth and systematic investigations using simulated or artificially constructed landscapes. Therefore, this study was focused on data sets from real landscapes, while our results from simulated landscapes, which generally support findings here, 
will be reported elsewhere. We also note that, although two general types of methods have been used in landscape pattern analysis - spatial statistics (including geostatistics) and pattern metrics, this paper deals only with the second. Excellent reviews on spatial statistical methods and their applications can be found in Rossi et al. (1992), Goovaerts (1997), and Fortin (1999). This study was designed to address the following specific questions: (1) How do changing grain size and changing extent affect different landscape metrics for a given landscape? (2) How does the behavior of various landscape metrics differ among distinctive landscapes? (3) Are there general scaling relations for certain landscape metrics that are consistent across landscapes?

These questions need to be addressed using landscape metrics computed both at the entire landscape level (taking account of all patch types altogether) and at the class level (each patch type being considered separately). Although many of the landscapeand class-level metrics are mathematically similar, their physical meanings are usually quite distinct (see McGarigal and Marks 1995). While the landscapelevel metrics are synoptic measures of the landscape as a whole, the class-level metrics provide information on each patch (or land cover) type in the landscape, which is necessary for most ecological or planning considerations. Wu et al. (2003) examined 19 landscape-level metrics based on five landscape data sets ( 4 of them used here in this study). This papers focuses on 17 class-level metrics and compares the scaling relations at the levels of the individual patch type and the whole landscape.

\section{Data and methods}

Seventeen class-level landscape metrics were examined in this study: Class Area (CA), Percent of Landscape (CA\%), Number of Patches (NP), Patch Density (PD), Total Edge (TE), Edge Density (ED), Largest Patch Index (LPI), Mean Patch Size (MPS), Patch Size Standard Deviation (PSSD), Patch Size Coefficient of Variation (PSCV), Landscape Shape Index (LSI), Mean Patch Shape Index (MPSI), AreaWeighted Mean Shape Index (AWMSI), Double-Log Fractal Dimension (DLFD), Mean Patch Fractal Dimension (MPFD), Area Weighted Mean Patch Fractal Dimension (AWMFD), and Square Pixel (SqP). The software package, FRAGSTATS 2.0 (McGarigal and Marks 1995), was used to compute the selected land- scape metrics, with Square Pixel Index (Frohn 1998) being added to the package by modifying the source code.

Four land use and land cover maps with contrasting spatial patterns were used for this study: (A) a boreal forest landscape with 11 land use and land cover types including various forest stands, disturbed areas and water, (B) Minden landscape in the Great Basin, USA with 15 land use and land cover types including native arid plant communities, burned areas, and urban and agricultural land uses, (C) Washoe landscape in the Great Basin, USA with 11 land use and land cover types most of which were shrublands, and (D) Phoenix urban landscape with 24 land use and land cover types, dominated by various urban and agricultural land uses. The boreal forest landscape in Canada showed little human disturbance, the two Great Basin landscapes in Nevada exhibited moderate urbanization and cultivation, and the metropolitan Phoenix landscape was a highly urbanized environment. Land use and land cover types varied considerably across these landscapes in terms of both the number and the content of patch types. The spatial resolution of all data sets was 30 by 30 meters, and the spatial extent varied from $357 \mathrm{~km}^{2}(630 \times 630$ pixels) for the boreal landscape to $2025 \mathrm{~km}^{2}$ $(1500 \times 1500$ pixels $)$ for the Phoenix landscape. Details of these study areas have been given elsewhere (Wu et al. 2003; Luck and Wu 2002).

To investigate the effects of changing grain size, the spatial resolution of three landscape maps (Boreal, Minden, and Phoenix) was systematically changed from 1 by 1 to 100 by 100 pixels with the extent kept constant, which was consistent with Wu et al. (2003). As the grain size increased, data were aggregated following the majority rule, which is one of the most commonly used methods for aggregating categorical data in ecology and remote sensing. Each new map, with progressively larger grain size (e.g., $1 \times 1,2 \times 2$, $\ldots, 100 \times 100)$, was created by directly aggregating the original data set, instead of using a cumulative procedure that would introduce more errors. When the grain size could not wholly divide the number of rows or columns of the data set, the remainder of rows or columns at the edge were excluded from the new map. This omission of edge rows and columns did not seem to be a problem as long as the extent/grain ratio was sufficiently large. To investigate the effects of changing extent, we systematically increased the extent of the maps diagonally starting from the north- 
west corner while keeping the grain size constant $(\mathrm{Wu}$ et al. 2003).

\section{Results}

Scaling relations with respect to changing grain size

With changing grain size through spatial aggregation, the responses of the 17 class-level metrics fell into two general groups: metrics showing consistent scaling relations (Type I) and metrics showing unpredictable scaling behavior (Type II). The first group was further divided into those showing both consistent and robust scaling relations (Type $\mathrm{I}_{\mathrm{A}}$ ) and those showing consistent but less robust scaling relations (Type $\mathrm{I}_{\mathrm{B}}$ ). Note that the word "consistent" here refers to the consistence of scaling relations between different landscapes, whereas the word "robust" indicates the similarity of scaling relations between different patch types within the same landscape.

Figure 1 shows examples of how different metrics responded to changing grain size for three study landscapes in the form of scalograms, i.e., plots of landscape metrics against scale (grain size or extent). Table 1 is a summary of the scaling relations and their characteristics with respect to changing grain size. Of the 17 metrics, 5 belonged to Type $\mathrm{I}_{\mathrm{A}}$ : Number of Patches (NP), Patch Density (PD), Total Edge (TE), Edge Density (ED), and Landscape Shape Index (LSI); and 7 to Type $\mathrm{I}_{\mathrm{B}}$ : Largest Patch Index (LPI), Square Pixel Index (SqP), Mean Patch Size (MPS), Patch Size Standard Deviation (PSSD), Patch Size Coefficient of Variation (PSCV), Area-Weighted Mean Shape Index (AWMSI), and Area-Weighted Mean Fractal Dimension (AWMFD). Type $\mathrm{I}_{\mathrm{A}}$ metrics exhibited a power-law scaling relation which was highly consistent and robust over a range of scales, i.e.,

$$
y=a x^{b}, \quad a>0, b<0
$$

where $y$ is the value of a landscape metric, $a$ and $b$ are constants, and $\times$ is the grain size expressed as the number of pixels along a side.

Type $I_{B}$ metrics showed several different scaling relations with a consistent "global pattern" between different landscapes, but rather high "short-range variations" between different patch types which may, in part, have been caused by the low abundance of some patch types. Specifically, AWMSI, AWMFD, MPS, and PSCV followed a power-law scaling relation (Equation 1), PSSD a linear function, and LPI a logarithmic function of the form,

$$
y=a \ln x+b
$$

where $y$ is the value of a landscape metric, $a$ and $b$ are constants, and $x$ is the grain size expressed as the number of pixels along a side. SqP seemed to exhibit two forms of scaling relations: a linear decreasing function for dominant patch types and an exponential decay function for less abundant patch types. The simple parimeter/area ratio increased linearly for most land cover types (not shown in Figure 1).

Type II metrics included: Class Area (CA), Percent of Landscape (CA\%), Mean Patch Shape Index (MSI), Mean Patch Fractal Dimension (MPFD), and Double-Log Fractal Dimension (DLFD). The values of these indices varied unpredictably with changing grain size, resulting in response curves of various forms - relatively constant, monotonic changes, or fluctuations. This suggested that these metrics were highly sensitive to the specific patterns of the landscapes under study, and thus general scaling relations were not possible to derive.

Not all 17 metrics are shown in Figure 1 due to space limitation. Some metrics showed similar scalograms because of mathematical similarity. For example, $\mathrm{CA}$ and $\mathrm{CA}(\%)$ showed exactly the same pattern because $\mathrm{CA}(\%)=\mathrm{CA} / \mathrm{A}$, where $\mathrm{A}$ is the landscape area which is a constant in the case of changing grain size. For the same reason, NP and PD exhibited an identical scaling relation, and so did TE and ED. As discussed later, however, this apparently was not the case with changing extent. On the other hand, MSI and MPFD exhibited similar patterns in response to both changing grain size and extent because of their mathematical similarity:

$$
\frac{M S I}{M P F D}=\sum_{i=1}^{N}\left(\frac{0.25 P_{i}}{\sqrt{a_{i}}}\right) / \sum_{i=1}^{N}\left(\frac{\ln \left(0.25 P_{i}\right)}{\ln \sqrt{a_{i}}}\right)
$$

That is, while MSI is simply a perimeter-area ratio normalized based on the square shape and averaged over all patches, MPDF requires that both the numerator and denominator be log-transformed before the summation for the entire class across the landscape. However, the range of change for MPDF was rather small (usually $<0.05$ and not exceeding 
(A) Boreal Landscape
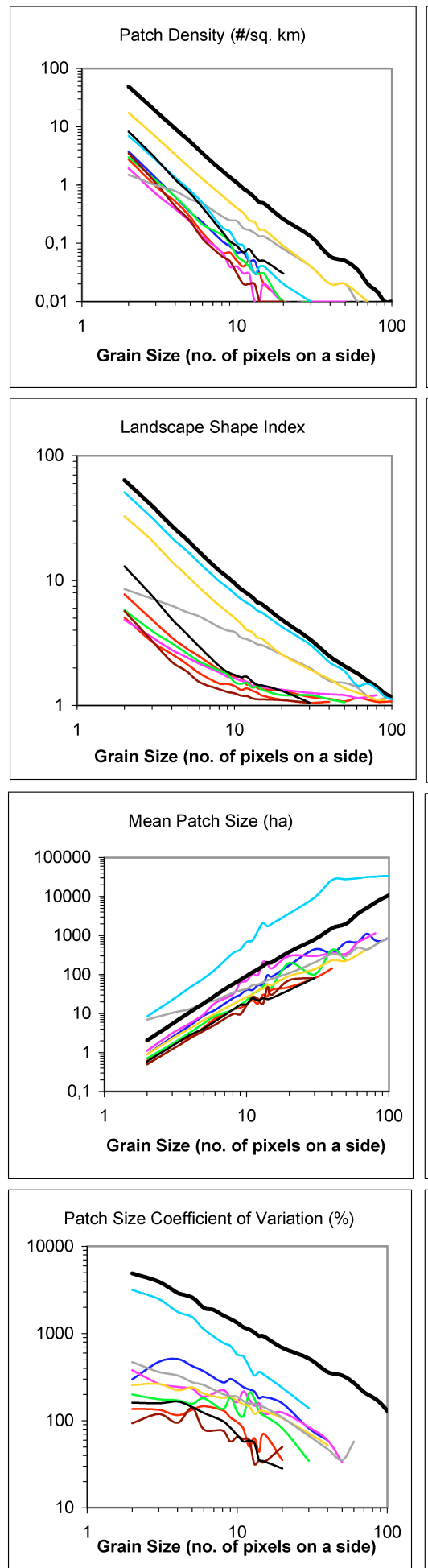

(B) Minden Landscape
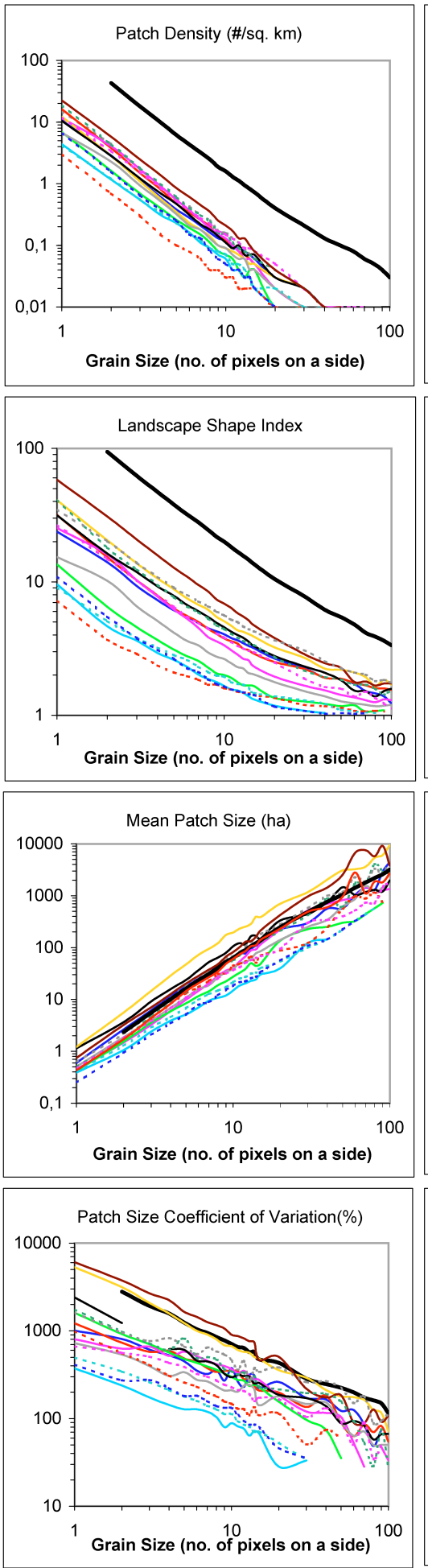

(C) Phoenix Landscape
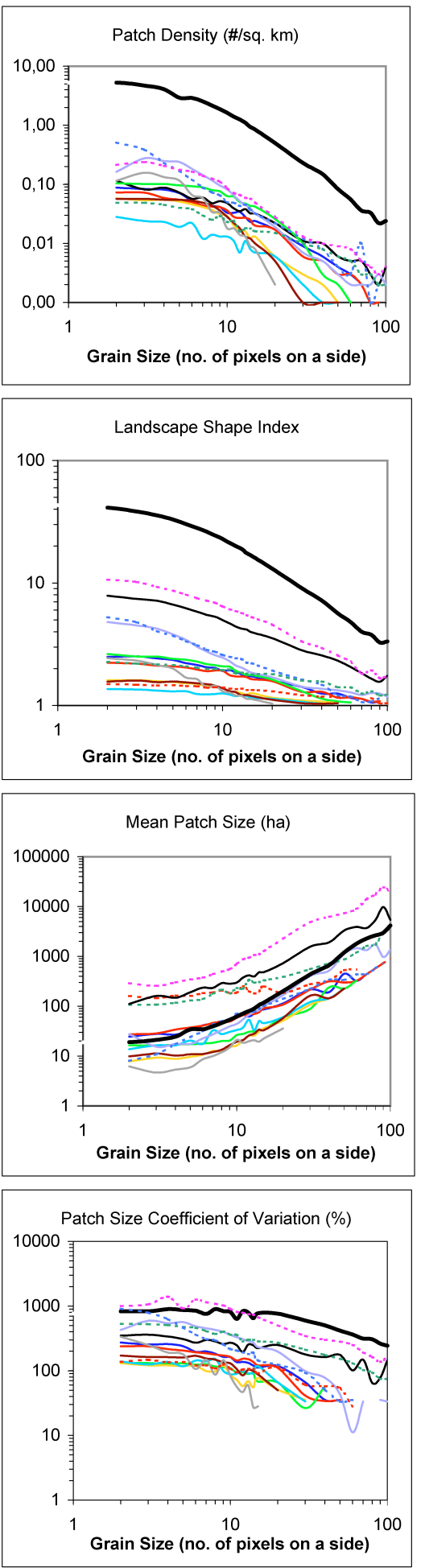

Figure 1. Examples of landscape metric scalograms with changing grain size: (A) a boreal forest landscape, (B) the Minden landscape, and (C) the central Phoenix urban landscape. The lines in each scalogram represent different patch types (i.e., land use and land cover types). Landscape-level metrics (the thick black lines) are also plotted for comparison. 
Table 1. Scaling relations of class-level metrics with respect to grainsize.

$\begin{array}{lc}\mathrm{I}_{\mathrm{A}} \text {. Metrics showing consistent and robust scaling relations } \\ \text { Number of Patches (NP) } & \text { Edge Density (ED) } \\ \text { Patch Density (PD) } & \text { Landscape Shape Index (LSI) } \\ \text { Total Edge (TE) } & \end{array}$

Total Edge (TE)

Characteristics of the scaling relations:

Power law:

$y=a x^{b}, \quad a>0, \quad b<0$

where $y$ is the value of a metric, $a$ and $b$ are constants, and $\times$ is the grain size expressed as the number of pixels along a side.

The scaling relation is consistent between patch types within the same landscape as well as among different landscapes.

$\mathrm{I}_{\mathrm{B}}$. Metrics showing consistent, but less robust scaling relations

Largest Patch Index (LPI) Patch Size Standard Deviation (PSSD)

Square Pixel Index (SqP) Patch Size Coefficient of Variation (PSCV)

Mean Patch Size (MPS) Area-Weighted Mean Shape Index (AWMSI)

Area-Weighted Mean Fractal Dimension (AWMFD)

Characteristics of the scaling relations:

Power law $\left(y=a x^{b}\right)$ : AWMSI, AWMFD, MPS, PSCV

Linear function $(y=a x+b)$ : PSSD

Logarithmic function $(y=a \ln x+b)$ : LPI

Linear or exponential decay: SqP

The scaling relations tend to be consistent among different landscapes, but the variability between patch types within the same landscape

is much greater than Type $\mathrm{I}_{\mathrm{A}}$ metrics. This between-patch type variability tends to decrease with the dominance of patch types.

II. Metrics exhibiting no consistent scaling relations

Class Area (CA)

Mean Patch Fractal Dimension (MPFD)

Percent of Landscape (CA\%)

Double-Log Fractal Dimension (DLFD)

Mean Patch Shape Index (MSI)

Characteristics of the scaling relations:

No consistent scaling relations among different landscapes.

Response curves may take various forms: relatively constant, monotonic changes, or fluctuations. Response curves are sensitive to the specific pattern of the landscape, and thus do not show consistent patterns across different landscapes.

0.1 ) in both cases of changing grain size and extent, which made it less desirable for comparison. Similarly, the scale response curves of AWMSI and AWMFD resembled each other because:

$$
\begin{aligned}
A W M S I / A W M F D= & \sum_{i=1}^{N}\left[\left(\frac{0.25 P_{i}}{\sqrt{a_{i}}}\right)\right. \\
& \left.\left(\frac{a_{i}}{A}\right)\right] / \sum_{i=1}^{N}\left(\frac{\ln \left(0.25 P_{i}\right)}{\ln \sqrt{a_{i}}}\left(\frac{a_{i}}{A}\right)\right)
\end{aligned}
$$

In this case, AWMFD seemed more preferable because it was bale to suppress somewhat the abrupt large fluctuations that occurred with AWMSI, so that a comparison between patch types became more feasible.

\section{Scaling relations with respect to changing extent}

The responses of class-level metrics to changing extent could also be classified into two groups: Type I metrics showing consistent and relatively robust scaling relations and Type II metrics with unpredictable scaling behavior (Table 2 and Figure 2). Type I metrics included NP, TE, LSI, SqP, and CA, whereas the other 12 belonged to Type II. NP, TE, and CA exhibited a power law scaling relation that was consistent between different landscapes but varied noticeably between patch types within the same landscape. With increasing extent, SqP increased rapidly initially and then began to approach a maximum value, whereas LSI tended to increase continuously. For relatively abundant patch types, a logarithmic function for $\mathrm{SqP}$ and a linear scaling function for LSI could be obtained by regression. These trends seemed consis- 
Table 2. Scaling relations of class-level metrics with respect to extent.

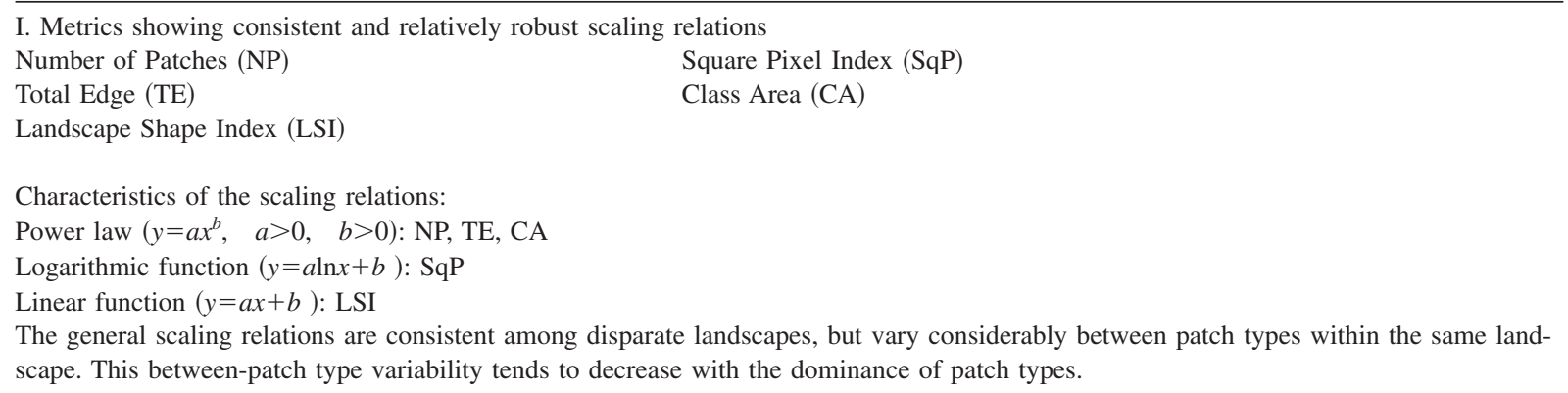

II. Metrics showing no consistent scaling relations

Percent of Landscape (CA\%)

Patch Size Coefficient of Variation (PSCV)

Patch Density (PD)

Edge Density (ED)

Mean Patch Size (MPS)

Largest Patch Index (LPI)

Patch Size Standard Deviation (PSSD)

Mean Patch Shape Index (MSI)

Area-Weighted Mean Shape Index (AWMSI)

Mean Patch Fractal Dimension (MPFD)

Double-Log Fractal Dimension (DLFD)

Area-Weighted Mean Fractal Dimension (AWMFD)

Characteristics of scaling relations:

No consistent scaling relations among different landscapes.

Response curves may take various forms: relatively constant, monotonic changes, or fluctuations.

Response curves are sensitive to the specific pattern of the landscape, and do not show consistent patterns across different landscapes.

tent among different landscapes, and the variability between patch types tended to decrease with the increasing abundance of patch types. The response curves of Type II metrics showed no consistent scaling patterns, and were highly dependent upon the specifics of the landscapes.

Overall, the effects of changing extent on landscape metrics were much less predictable than those of changing grain size. This was evident from two facts: (1) the number of Type I metrics was much smaller for changing extent than for changing grain size (5 vs. 12); and (2) Type I metrics for grain size were less variable than those for extent between patch types and among landscapes. Several differences between changing grain size and extent are noteworthy. Unlike in the case of changing grain size, PD and ED did not show any consistent scaling functions although their behavioral patterns seemed to resemble each other. As grain size increased, PSSD tended to increase linearly, while PSCV declined and MPS increased both in a power-law fashion. In contrast, as extent increased, MPS changed unpredictably, but with a much smaller magnitude than in the case of changing grain size. Thus, PSSD and PSCV both tended to increase in a similar way for most land cover types $(P S C V=P S S D / M P S)$. This was most obvious for the boreal landscape, in which the behav- ioral patterns of PSSD and PSCV resembled each other closely because MPS changed little over the entire range of extent.

Because $L S I=0.25 T E \sqrt{T A}$ (where $T A$ is the total area of the landscape), $L S I$ and $T E$ behaved the same way in response to changing grain size. However, in the case of changing extent, if $T E$ increases as a power function $\left(y=a x^{b}\right)$ with extent (measured as the number of pixels on a side), LSI should follow a scaling function of the form, $y \propto x^{b-1}$. Then, if $b$ is close to 2, then LSI should behave nearly linearly. Our results indeed showed that for most patch types LSI tended to increase linearly, but with considerable variations. The deviations of the observed patterns for LSI from a linear function were attributable to the considerably variable scale responses of TE. Also, LSI and SqP are numerically related to each other, i.e., $L S I=(1-$ $S q P)^{-1}$. Thus, the response curves of LSI and SqP reflected this relationship. Both of them showed much greater variations, and thus less predictability, between patch types and among landscapes in the case of changing extent. Another interesting finding was that DLFD, for most patch types, was unpredictable and varied increasingly with increasing grain size, but appeared to be relatively constant with continuing increase in extent after initial fluctuations at smaller scales. The latter was reminiscent of the notion that 
(A) Boreal Landscape
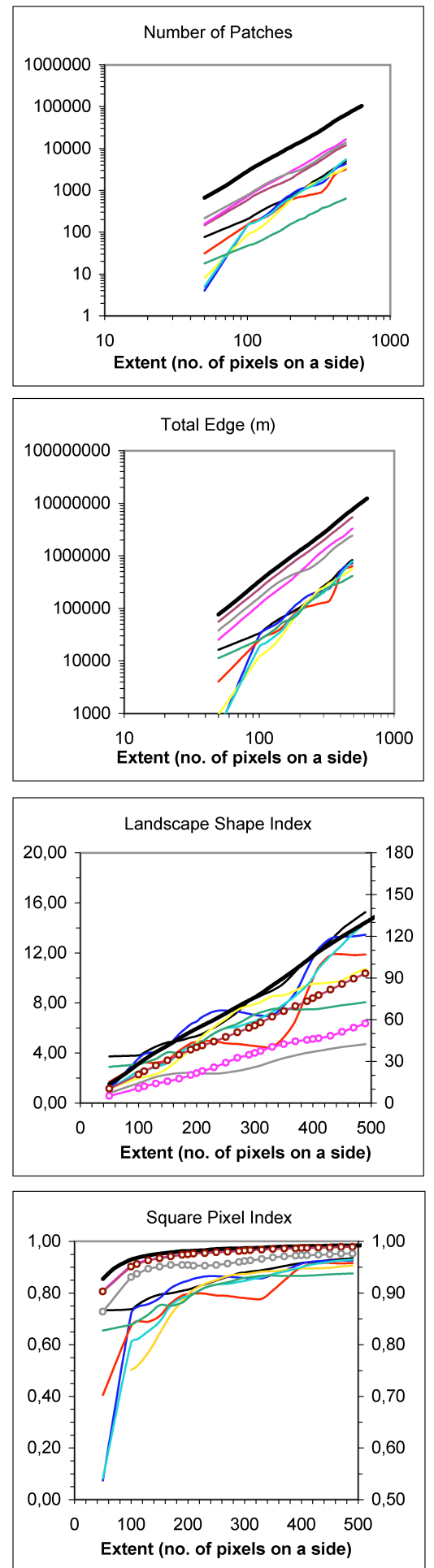

(B) Minden Landscape
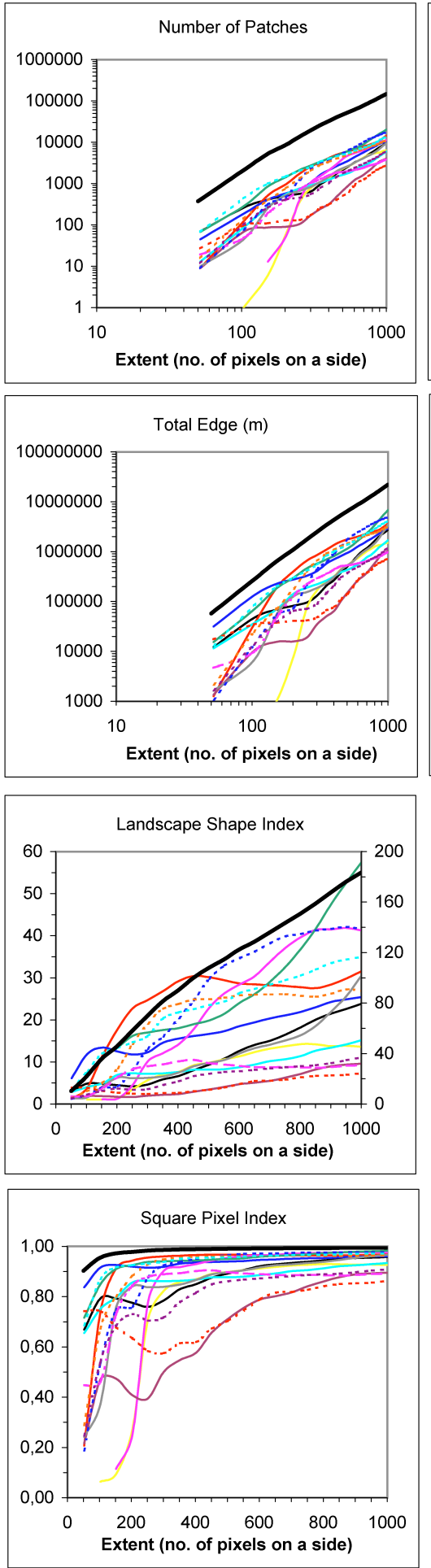

(C) Phoenix Landscape
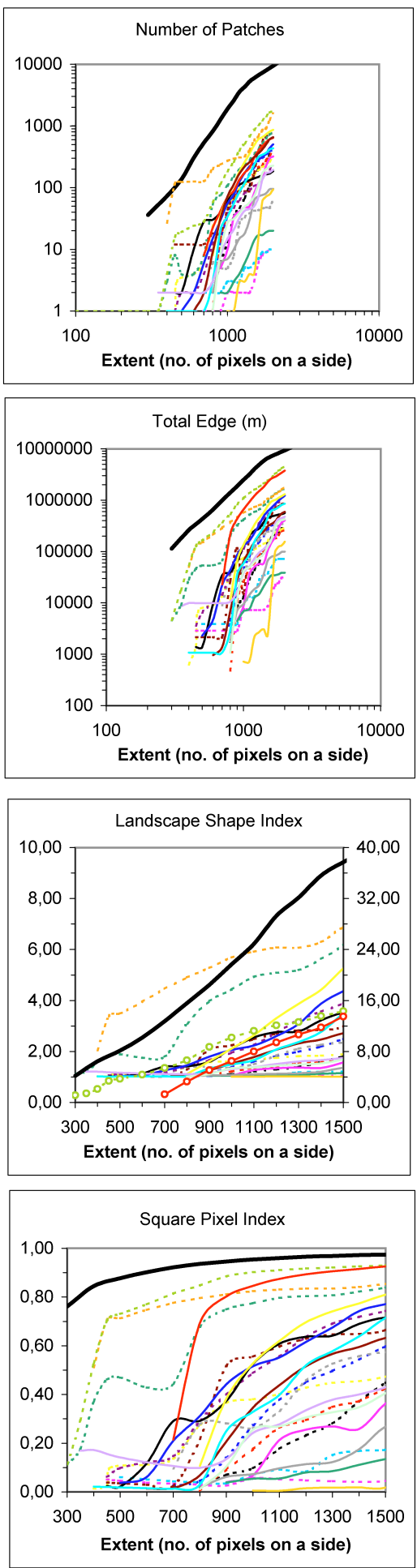

Figure 2. Examples of landscape metric scalograms with changing extent: (A) a boreal forest landscape, (B) the Minden landscape, (C) the central Phoenix urban landscape (Washoe landscape exhibited similar patterns, but was not shown here due to space limitation). The lines in each scalogram represent different patch types (i.e., land use and land cover types). Landscape-level metrics (the thick black lines) are also plotted for comparison. Note that for the ease of visualization, the second y-axis is used for landscape-level metrics and individual patch types (lines with open circles) whose values are too large or too small as compared to the rest of the patch types (the bottom row). 
Table 3. Comparison of scaling relations of class- and landscape-level metrics.

\begin{tabular}{|c|c|c|c|c|}
\hline \multirow[b]{3}{*}{ Landscape Metrics } & \multicolumn{4}{|c|}{ Types of Scaling Relations } \\
\hline & \multicolumn{2}{|l|}{ Grain Size } & \multicolumn{2}{|l|}{ Extent } \\
\hline & Class-Level & Landscape-Level & Class-Level & Landscape-Level \\
\hline Number of Patches & power law & power law & power law & power law \\
\hline Patch Density & power law & power law & unpredictable & unpredictable \\
\hline Total Edge & power law & power law & power law & power law \\
\hline Edge Density & power law & power law & unpredictable & unpredictable \\
\hline Landscape Shape Index & power law & power law & linear & linear \\
\hline Area-Weighted Mean Shape Index & power law & power law & unpredictable & staircase \\
\hline Area-Weighted Mean Fractal Dimension & power law & power law & unpredictable & staircase \\
\hline Mean Patch Size & power law & power law & unpredictable & unpredictable \\
\hline Patch Size Coefficient of Variation & power law & power law & unpredictable & staircase \\
\hline Patch Size Standard Deviation & linear & linear & unpredictable & staircase \\
\hline Square Pixel & linear or exponential & linear & logarithmic & $\begin{array}{l}\text { logarithmic or power } \\
\text { law }\end{array}$ \\
\hline Largest Patch Index & logarithmic & $\begin{array}{l}\text { logarithmic or power } \\
\text { law }\end{array}$ & unpredictable & unpredictable \\
\hline Mean Patch Shape Index & no scaling relation & no scaling relation & unpredictable & unpredictable \\
\hline Double-Log Fractal Dimension & unpredictable & unpredictable & unpredictable & unpredictable \\
\hline Mean Patch Fractal Dimension & unpredictable & unpredictable & unpredictable & unpredictable \\
\hline Patch Richness & $\mathrm{n} / \mathrm{a}$ & staircase & $\mathrm{n} / \mathrm{a}$ & staircase \\
\hline Patch Richness Density & $\mathrm{n} / \mathrm{a}$ & staircase & $\mathrm{n} / \mathrm{a}$ & power law \\
\hline Shannon's Diversity Index & $\mathrm{n} / \mathrm{a}$ & staircase & $\mathrm{n} / \mathrm{a}$ & logarithmic \\
\hline Contagion & $\mathrm{n} / \mathrm{a}$ & unpredictable & $\mathrm{n} / \mathrm{a}$ & unpredictable \\
\hline Class Area & unpredictable & $\mathrm{n} / \mathrm{a}$ & power law & $\mathrm{n} / \mathrm{a}$ \\
\hline Percent of Landscape & unpredictable & $\mathrm{n} / \mathrm{a}$ & $\begin{array}{l}\text { linear or power } \\
\text { law }\end{array}$ & $\mathrm{n} / \mathrm{a}$ \\
\hline
\end{tabular}

landscapes may exhibit self-similarity over a finite range of spatial extent (Milne 1991; Milne 1992; Lam and Quatrochi 1992).

\section{Discussion}

Comparing scaling relations of class- and landscape-level metrics

In a related study, Wu et al. (2003) showed that the responses of 19 landscape-level metrics to changing grain size and extent could be categorized as three general types: (1) Type I metrics exhibiting consistent and robust scaling relations in the forms of linear, power, or logarithmic functions over a range of scales; (2) Type II metrics showing staircase-like responses with changing scale; and (3) Type III metrics behaving erratically in response to changing scale and with no consistent scaling relations among different landscapes. In the case of changing grain size, 12 metrics belonged to Type I: NP, PD, TE, ED, LSI, AWMSI, AWMFD, PSCV, MPS, SqP, PSSD, and
LPI; 3 to Type II: PR (Patch Richness), PRD (Patch Richness Density), and SHDI (Shannon's Diversity Index); and 4 to Type III: DLFD, CONT (Contagion), MPFD, and MSI. In the case of changing extent, the number of Type I metrics reduced to 6: NP, TE, SqP, PRD, SHDI, and LSI; the number of Type II metrics was 5: PR, PSSD, PSCV, AWMSI, and AWMFD; and the number of Type III metrics increased to 8: PD, ED, DLFD, MPS, LPI, CONT, MSI, and MPFD. In addition, Wu et al. (2003) showed that the starting position and the direction of changing extent could also significantly influence the scaling patterns for certain landscape metrics.

In this study, four landscape-level metrics (PR, PRD, SHDI, and CONT), not applicable at the level of individual patch types, were not used, and the absolute and relative areas of each patch type (i.e., class area, CA and percent of landscape, CA\%) were added. By comparing the class-level curves (thin lines) with the landscape-level curve (thick line) in each landscape metric scalogram in Figure 1, Figure 2 , the similarities and differences between them become apparent. Table 3 is a summary of the compari- 
son of scaling relations between the class and landscape levels. The same 15 metrics used at both the class and landscape levels showed rather similar scaling patterns in terms of both the specific metrics and the scaling relations. In general, effects of changing grain size were more predictable than changing extent in that more metrics showed consistent scaling relations across different landscapes in the former case. However, it must be emphasized here that artifacts due to data aggregation and consequent analysis may become overwhelming for classes with extremely low abundance. Because of the high betweenpatch type variability at the class level, the staircaselike responses of such metrics as AWMSI, AWMFD, PSCV, and PSSD with respect to changing extent appeared consistent only at the landscape level.

It is important to note that this study only used one of the several methods of aggregating spatial data the majority rule method. Although this may be the most commonly used one in ecological and remote sensing applications, it would be interesting to compare how different aggregation methods affect the characteristics of landscape metric scalograms. A number of studies have shown that different aggregation methods may have significant effects on spatial model evaluation, land cover classification, and landscape pattern analysis (Costanza 1989; Justice et al. 1989; Bian and Butler 1999; Turner et al. 2001). Thus, aggregation methods may also affect scaling relations of landscape metrics.

\section{Scale multiplicity of landscapes and multiscale pattern analysis}

The relationship between pattern and scale has been a central issue in ecology and geography (MacArthur 1972; Meentemeyer 1989; Levin 1992; Wu and Loucks 1995). Pattern is rooted in spatial heterogeneity which in turn stems from variations of spatial dependence. The first law in geography - "Everything is related to everything else, but near things are more related than distant things" (sensu Tobler 1970) - is essentially a law of spatial autocorrelation. However, heterogeneity manifests itself as patchiness and gradients intertwined over a wide range of spatial scales. Thus, scale dependence is as essential a property of heterogeneity as is spatial dependence. This has led to the claim of "the second law in geography" (sensu Arbia et al. 1996): "Everything is related to everything else, but things observed at a coarse spatial resolution are more related than things observed at a finer resolution." This is simply a law of scale dependence of correlation, which was developed based on both empirical and analytical results that the correlation between variables increases while variance decreases as the resolution (grain size) of spatial data is increased (Amrhein 1995; Arbia et al. 1996; Wu et al. 2000). Of course, not only are correlation coefficients and variance scale dependent, so are a variety of landscape indices, statistical methods, and mathematical models as well.

Many, if not most, landscapes are hierarchically structured (Urban et al. 1987; Woodcock and Harward 1992; Wu and Loucks 1995; Reynolds and Wu 1999; Wu 1999). Even before hierarchy theory became an influential perspective in ecology and before multi-scale pattern analyses became commonly practiced in earth sciences, the eminent ecologist Robert MacArthur (1972) already unambiguously recognized the scale multiplicity and hierarchical nature of landscapes, as he said: "A real environment has a hierarchical structure. That is to say, it is like a checkerboard of habitats, each square of which has, on closer examination, its own checkerboard structure of component subhabitats. And even the tiny squares of these component checkerboards are revealed as themselves checkerboards, and so on. All environments have this kind of complexity, but not all have equal amounts of it."

To understand the structure and functioning of landscapes, therefore, the scale dependence of spatial heterogeneity must be quantified. Recent developments in landscape ecology have provided a new impetus as well as a suite of innovative theories and methods for achieving this goal (Turner et al. 2001; $\mathrm{Wu}$ and Hobbs 2002). To identify the characteristic scales or hierarchical levels of landscape structure, two general approaches are available. The first is to use statistical methods that are inherently multiscaled, such as semivariance analysis (Burrough 1995), wavelet analysis (Bradshaw and Spies 1992), spectral analysis (Platt and Denman 1975), fractal analysis (Milne 1997), lacunarity analysis (Plotnick et al. 1993), and scale variance (Moellering and Tobler 1972; Wu et al. 2000). The second is, as illustrated in this study, to construct scalograms using simple measures such as landscape metrics computed progressively over a series of scales and then to explore scaling functions. Spatial statistical methods have been known for their ability to detect characteristic scales, and, in particular, semivariance analysis has been frequently used for this purpose. However, re- 
cent studies have indicated that in semivariograms of real landscapes fine-scale variability can be "squeezed" by broad-scale variability, so that multiscale structure may be obscured (Meisel and Turner 1998; Wu et al. 2000). In addition, the results of semivariance analysis and their interpretations can also be significantly affected by changing the grain size, lag, and extent of the data sets (Dungan et al. 2002).

Can landscape metrics detect hierarchical structures of landscapes when repeatedly computed at multiple scales? Apparently, not all of them can. Our earlier study showed that progressively increasing extent allowed certain landscape metrics (e.g., PSCV, PSSD, MPS, AWMSI, AWMFD) to reflect some dramatic shifts in the average properties of the landscape concerning the size and shape of patches (Table 3; Wu et al. 2003). Other studies also demonstrated that simple variance and correlation measures were able to detect scale breaks in real and artificial landscapes when calculated at different grain sizes (e.g., O'Neill et al. 1991; Wu et al. 2000). Nevertheless, there are at least three reasons for the lack of scale breaks in the landscape metric scalograms in this and other similar studies. First, when grain size is increased always in a square shape following the majority rule, the hierarchical structure in real landscapes may be distorted or masked because of the high variability in patch size, shape and orientation (Wu et al. 2000). This problem may be ameliorated by a "patch-based" or "object-specific" aggregation scheme (Hay et al. 2001). Second, different landscape metrics represent different aspects of landscape structure, and for a given landscape not all of them exhibit hierarchical structure although they may all be scale-dependent. Third, even for landscape attributes that do posses hierarchical structure, be it structural or functional, they may form multiple hierarchies in the same landscape which may not correspond to each other precisely in terms of spatial and temporal scales (Wu 1999). While the first reason is primarily methodological, the last two involve both the theory of hierarchical systems and the understanding of the systems under study.

\section{Spatial allometry and landscape pattern}

It is intriguing that several metrics were found to exhibit power scaling relationships which were ostensibly consistent between different classes within the same landscape and among different landscapes.
Other measures of landscape pattern that were not considered in this study may also scale spatially in a power law fashion. For example, Costanza and Maxwell (1994) found that with increasing spatial resolution (i.e., decreasing grain size), the spatial autopredictability (the reduction in uncertainty about the state of a pixel in a scene given knowledge of the sate of adjacent pixels in that scene) increases and spatial cross-predictability (the reduction in uncertainty about the state of a pixel in a scene given knowledge of the state of corresponding pixels in other scenes) decreases both linearly on a log-log scale. From this study, it is evident that power-law scaling is more often and more consistent in the case of changing grain size than changing extent. It is attempting to relate these landscape metric scaling relations to other power laws in biological and ecological systems particularly, body-size allometry (biological attributes scale with body size following a power law; e.g., Brown et al. 2000) and spatial allometry (ecological attributes scale with ecosystem size or spatial extent following a power function; e.g., Schneider 2000). The power, elegance, and mystery of allometric scaling all lie in the simple equation, $y=a x^{b}$, where the dependent variable $y$ is a biological or an ecological variable of interest, $\times$ is mostly body size in traditional biological allometry or a spatial scale measure (e.g., grain, extent, or their ratio), $a$ is the normalization constant, and $b$ is the scaling exponent. Thus, the power laws of landscape metrics may be considered as an extension of spatial allometry.

Do the power scaling relations in this study necessarily imply that a single underlying process is responsible for the landscape attributes these metrics represent? Our knowledge of these rather different landscapes suggests that a number of natural and anthropogenic processes, operating on distinctive spatial and temporal scales, have generated these landscape patterns. Does this, then, suggest that multiplicative processes can give rise to seemingly scale-invariant landscape patterns with simple allometric scaling relations and no characteristic scales? These are certainly intriguing questions that still await future research. Although power laws have frequently been related directly to, or cast in the light of, self-similarity, scale-invariance, and universality, such interpretations need to be made with caution. Power laws may arise from different processes, either internal or external to the system under consideration (Jensen 1998; Plotnick and Sepkoski 2001), and they usually 
hold only over a finite ranges of spatiotemporal scales (Wiens 1989; Milne 1991; Milne 1992; Lam and Quatrochi 1992; Wu 1999). More in-depth discussions on these issues as well as other scaling theories and methods are given in Wu et al. (2004).

\section{Conclusions}

Landscape metrics have been widely used in ecological and geographical studies and provided valuable insight into the structural characteristics of complex landscapes. However, the lack of a comprehensive understanding of the scale sensitivity of these metrics seriously undermines their interpretation and usefulness. This study has systematically investigated how landscape metrics respond to changing grain size and extent, allowing for exploration of general scaling relations and idiosyncratic behaviors.

The results of this study showed that changing grain size and extent had significant effects on both the class- and landscape-level metrics. Although the landscapes under study were quite different in both the composition and configuration of patches, the effects of changing scale fell into two categories (simple scaling functions and unpredictable) for the class-level metrics, and three categories for the landscape-level metrics (simple scaling functions, staircase-like scaling behavior, and unpredictable). Overall, more metrics showed consistent scaling relations with changing grain size than with changing extent at both the class and landscape levels - indicating that effects of changing spatial resolution are generally more predictable than those of changing map sizes. While the same metrics tended to behave similarly at the class level and the landscape level, the scale responses at the class level were much more variable. These results appear robust not only across different landscapes, but also independent of specific map classification schemes.

This study corroborates the increasingly recognized notions: there is no single "correct" or "optimal" scale for characterizing spatial heterogeneity, and comparison between landscapes using pattern indices must be based on the same spatial resolution and extent. In addition, these results may provide practical guidelines for scaling of spatial pattern. For example, landscape metrics with simple scaling relations reflect those landscape features that can be extrapolated or interpolated across spatial scales readily and accurately using only a few data points. In con- trast, unpredictable metrics represent landscape features whose extrapolation is difficult, which requires information on the specifics of the landscape of concern at many different scales. Finally, to quantify spatial heterogeneity using landscape metrics, it is both necessary and desirable to use landscape metric scalograms, in stead of single-scale values. Indeed, a comprehensive empirical database containing pattern metric scalograms and other forms of multiple-scale information of diverse landscapes is crucial for achieving a general understanding of landscape patterns and developing spatial scaling rules.

\section{Acknowledgments}

I would like to thank C. Overton, W. Shen, L. Zhang and M. A. Luck for their assistance with data preparation and analysis. Comments from three anonymous reviewers improved the paper and were greatly appreciated. This research was supported in part by grants from the United States Department of Agriculture (USDA-NRICGP 95-37101-2028), the U.S. Environmental Protection Agency's Science to Achieve Results program (R827676-01-0), and US National Science Foundation (DEB 97-14833, CAP-LTER).

\section{References}

Allen T.F.H. and Starr T.B. 1982. Hierarchy: Perspectives for Ecological Complexity. University of Chicago Press, Chicago, Illinois, USA.

Allen R.F.H., O'Neill R.V. and Hoekstra T.W. 1984. Interlevel relations in ecological research and management. USDA Forest Service Gen. Tech. Rep. RM-110, Rocky Mountain Forest and Range Experiment Station.

Amrhein C.G. 1995. Searching for the elusive aggregation effect: evidence from statistical simulations. Environment and Planning A 27: 105-119.

Arbia G., Benedetti R. and Espa G. 1996. Effects of the MAUP on image classification. Geogr. Syst. 3: 123-141.

Benson B.J. and Mackenzie M.D. 1995. Effects of sensor spatial resoltuion on landscape structure parameters. Landscape Ecology 10: 113-120.

Bian L. and Walsh S.J. 1993. Scale dependencies of vegetation and topography in a mountainous environment of Montana. Professional Geographer 45: 1-11.

Bian L. and Butler R. 1999. Comparing effects of aggregation methods on statistical and spatial properties of simulated spatial data. Photogrammatic Engineering and Remote Sensing 65: 7384.

Bradshaw G.A. and Spies T.A. 1992. Characterizing canopy gap structure in forests using wavelet analysis. Journal of Ecology 80: 205-215. 
Brown J.H. and West G.B. (ed.), 2000. Scaling in Biology. Oxford University Press, New York, New York, USA.

Burrough P.A. 1995. Spatial aspects of ecological data. In: Jongman R.H.G., Ter Braak C.J.F. and Van Tongeren O.F.R. (eds), Data Analysis in Community and Landscape Ecology. pp. 213265. Cambridge University Press, Cambridge, UK.

Costanza R. 1989. Model goodness of fit - A multiple resolution procedure. Ecological Modelling 47: 199-215.

Costanza R. and Maxwell T. 1994. Resolution and predictability: An approach to the scaling problem. Landscape Ecology 9: 4757.

Dale M.R.T. 1999. Spatial Pattern Analysis in Plant Ecology. Cambridge University Press, Cambridge, UK.

Dungan J.L., Perry J.N., Dale M.R.T., Legendre P., Citron-Pousty S., Fortin M.-J., Jakomulska A., Miriti M. and Rosenberg M.S. 2002. A balanced view of scale in spatial statistical analysis. Ecography 25: 626-640.

Fortin M.J. 1999. Spatial statistics in landscape ecology. In: Klopatek J.M. and Gardner R.H. (eds), Landscape Ecological Analysis. pp. 253-279. Springer-Verlag, New York, New York, USA.

Frohn R.C. 1998. Remote Sensing for Landscape Ecology. Lewis Publishers, Boca Raton, Florida, USA.

Gardner R.H., Milne B.T., Turner M.G. and O'Neill R.V. 1987. Neutral models for the analysis of broad-scale landscape pattern. Landscape Ecology 1: 19-28.

Goovaerts P. 1997. Geostatistics for Natural Resources Evaluation. Oxford University Press, New York, New York, USA.

Gustafson E.J. 1998. Quantifying landscape spatial pattern: What is the state of the art? Ecosystems 1: 143-156.

Hargis C.D., Bissonette J.A. and David J.L. 1998. The behavior of landscape metrics commonly used in the study of habitat fragmentation. Landscape Ecology 13: 167-186.

Hay G., Marceau D.J., Dubé P. and Bouchard A. 2001. A multiscale framework for landscape analysis: Object-specific analysis and upscaling. Landscape Ecology 16: 471-490.

Jelinski D.E. and Wu J. 1996. The modifiable areal unit problem and implications for landscape ecology. Landscape Ecology 11: $129-140$

Jensen H.J. 1998. Self-Organized Criticality: Emergent Complex Behavior in Physical and Biological Systems. Cambridge University Press, New York, New York, USA.

Justice C.O., Markham B.L., Townshend J.R.G. and Kennard R.L. 1989. Spatial degradation of satellite data. International Journal of Remote Sensing 10: 1539-1561.

Keeling M.J., Mezic I., Hendry R.J., McGlade J. and Rand D.A. 1997. Characteristic length scales of spatial models in ecology via fluctuation analysis. Philosophical Transactions of the Royal Society (London B) 352: 1589-1601.

King A.W., Johnson A.R. and O'Neill R.V. 1991. Transmutation and functional representation of heterogeneous landscapes. Landscape Ecology 5: 239-253.

Lam N.S.-N. and Quattrochi D.A. 1992. On the issues of scale, resolution, and fractal analysis in the mapping sciences. Professional Geographer 44: 88-98.

Levin S.A. 1992. The problem of pattern and scale in ecology. Ecology 73: 1943-1967.

Luck M. and Wu J. 2002. A gradient analysis of urban landscape pattern: A case study from the Phoenix metropolitan region, Arizona, USA. Landscape Ecology 17: 327-339.
MacArthur R.H. 1972. Geographical Ecology: Patterns in the Distribution of Species. Princeton University Press, Princeton, New Jersey, USA.

Marceau D.J. 1999. The scale issue in social and natural sciences. Canadian Journal of Remote Sensing 25: 347-356.

McGarigal K. and Marks B.J. 1995. FRAGSTATS: Spatial Pattern Analysis Program for Quantifying Landscape Structure. Gen. Tech. Rep. PNW-GTR-351. Pacific Northwest research Station. Meentemeyer V. and Box E.O. 1987. Scale effects in landscape studies. In: Turner M.G. (ed.), Landscape Heterogeneity and Disturbance. pp. 15-34. Springer-Verlag, New York, New York, USA.

Meentemeyer V. 1989. Geographical perspectives of space, time, and scale. Landscape Ecology 3: 163-173.

Meisel J.E. and Turner M.G. 1998. Scale detection in real and artificial landscapes using semivariance analysis. Landscape Ecology 13: 347-362.

Milne B.T. 1991. Heterogeneity as a multiscale characteristic of landscapes. In: Kolasa J. and Pickett S.T.A. (eds), Ecological Heterogeneity. pp. 69-84. Springer-Verlag, New York, New York, USA.

Milne B.T. 1992. Spatial aggregation and neutral models in fractal landscapes. American Naturalist 139: 32-57.

Milne B.T. 1997. Applications of fractal geometry in wildlife biology. In: Bissonette J.A. (ed.), Wildlife and Landscape Ecology: Effects of Pattern and Scale. pp. 32-69. Springer-Verlag, New York, New York, USA.

Moellering H. and Tobler W. 1972. Geographical variances. Geographical Analysis 4: 34-64.

Moody A. and Woodcock C.E. 1994. Scale-dependent errors in the estimation of land-cover proportions: Implications for global land-cover datasets. Photogrammatic Engineering and Remote Sensing 60: 585-594.

O’Neill R.V. 1979. Transmutations across hierarchical levels. In: Innis G.S. and O'Neill R.V. (eds), Systems Analysis of Ecosystems. pp. 59-78. International Co-operative, Fairland, Maryland, USA.

O'Neill R.V., DeAngelis D.L., Waide J.B. and Allen T.F.H. 1986 A Hierarchical Concept of Ecosystems. Princeton University Press, Princeton, New Jersey, USA.

O’Neill R.V., Gardner R.H., Milne B.T., Turner M.G. and Jackson B. 1991. Heterogeneity and spatial hierarchies. In: Kolasa J. and Pickett S.T.A. (eds), Ecological Heterogeneity. pp. 85-96. Springer-Verlag, New York, New York, USA.

O'Neill R.V., Hunsaker C.T., Timmins S.P., Timmins B.L., Jackson K.B., Jones K.B., Riitters K.H. and Wickham J.D. 1996. Scale problems in reporting landscape pattern at the regional scale. Landscape Ecology 11: 169-180.

Openshaw S. 1984. The Modifiable Areal Unit Problem. Geo Books, Norwich, UK.

Platt T. and Denman K.L. 1975. Spectral analysis in ecology. Annual Review of Ecology \& Systematics 6: 189-210.

Plotnick R.E., Gardner R.H. and O'Neill R.V. 1993. Lacunarity indices as measures of landscape texture. Landscape Ecology 8: 201-211.

Plotnick R.E. and Sepkoski J.J. 2001. A multiplicative multifractal model for originations and extinctions. Paleobiology 27: 126-139.

Qi Y. and Wu J. 1996. Effects of changing spatial resolution on the results of landscape pattern analysis using spatial autocorrelation indices. Landscape Ecology 11: 39-49. 
Reynolds J.F. and Wu J. 1999. Do landscape structural and functional units exist? In: Tenhunen J.D. and Kabat P. (eds), Integrating Hydrology, Ecosystem Dynamics, and Biogeochemistry in Complex Landscapes. pp. 273-296. Wiley, Chichester, UK.

Robinson A.H. 1950. Ecological correlation and the behaviour of individuals. Am. Soc. Rev. 15: 351-357.

Rossi R.E., Mulla D.J., Journel A.G. and Franz E.H. 1992. Geostatistical tools for modeling and interpreting ecological spatial dependence. Ecological Monographs 62: 277-314.

Saura S. and Martinez-Millan J. 2001. Sensitivity of landscape pattern metrics to map spatial extent. Photogrammatic Engineering and Remote Sensing 67: 1027-1036.

Schneider D.C. 2001. Spatial allometry. In: Gardner R.H., Kemp W.M., Kennedy V.S. and Petersen J.E. (eds), Scaling Relations in Experimental Ecology. pp. 113-153. Columbia University Press, New York, New York, USA.

Schneider D.C. 2001. The rise of the concept of scale in ecology. BioScience 51: 545-553.

Tobler W. 1970. A computer movie simulating urban growth in the Detroit region. Econ. Geogr. (Suppl.) 46: 234-240.

Turner M.G., O’Neill R.V., Gardner R.H. and Milne B.T. 1989. Effects of changing spatial scale on the analysis of landscape pattern. Landscape Ecology 3: 153-162.

Turner S.J., O’Neill R.V., Conley W., Conley M.R. and Humphries H.C. 1991. Pattern and scale: Statistics for landscape ecology. In: Turner M.G. and Gardner R.H. (eds), Quantitative Methods in Landscape Ecology. pp. 17-49. Springer-Verlag, New York, New York, USA.

Turner M.G., Gardner R.H. and O’Neill R.V. 2001. Landscape Ecology in Theory and Practice: Pattern and Process. SpringerVerlag, New York, New York, USA

Urban D.L., O’Neill R.V. and Shugart H.H. 1987. Landscape ecology: A hierarchical perspective can help scientists understand spatial patterns. BioScience 37: 119-127.
Wickham J.D. and Riitters K.H. 1995. Sensitivity of landscape metrics to pixel size. International Journal of Remote Sensing 16: 3585-3595.

Wiens J.A. 1989. Spatial scaling in ecology. Functional Ecology 3: 385-397.

Woodcock C. and Harward V.J. 1992. Nested-hierarchical scene models and image segmentation. International Journal of Remote Sensing 13: 3167-3187.

Wrigley N., Holt T., Steel D. and Tranmer M. 1996. Analysing, modeling, and resolving the ecological fallacy. In: Longley P. and Batty M. (eds), Spatial Analysis: Modellign in a GIS Environment. pp. 23-40. GeoInformation International, Cambridge, UK.

Wu J. and Levin S.A. 1994. A spatial patch dynamic modeling approach to pattern and process in an annual grassland. Ecological Monographs 64(4): 447-464.

Wu J. and Loucks O.L. 1995. From balance-of-nature to hierarchical patch dynamics: A paradigm shift in ecology. Quarterly Review of Biology 70: 439-466.

Wu J. 1999. Hierarchy and scaling: Extrapolating information along a scaling ladder. Canadian Journal of Remote Sensing 25: 367-380.

Wu J., Jelinski D.E., Luck M. and Tueller P.T. 2000. Multiscale analysis of landscape heterogeneity: Scale variance and pattern metrics. Geogr. Info. Sci. 6: 6-19.

Wu J. and Hobbs R. 2002. Key issues and research priorities in landscape ecology: An idiosyncratic synthesis. Landscape Ecology 17: 355-365.

Wu J., Shen W., Sun W. and Tueller P.T. 2003. Empirical patterns of the effects of changing scale on landscape metrics. Landscape Ecology 17: 761-782.

Wu J., Jones B., Li H. and Loucks O. L. (ed.), 2004. Scaling and Uncertainty Analysis in Ecology. Columbia University Press, New York, New York, USA (in press). 